\title{
LOS FLUJOS EXTRAREGIONALES EN TRÁNSITO POR CENTROAMÉRICA: UNA REVISIÓN DE LITERATURA Y MIRADAS FUTURAS PARA COMPRENDER UN COMPLEJO FENÓMENO MIGRATORIO
}

\author{
Extraregional flows in transit through Central America: \\ a literature review and future perspectives to understand a complex \\ migratory phenomenon
}

\author{
Juan C. Méndez Barquero*
}

\begin{abstract}
Resumen. El artículo examina la complejidad teórico-conceptual de la migración en tránsito como unidad de análisis a partir del caso de estudio de los flujos migratorios extraregionales en tránsito por Centroamérica, en particular, a partir del año 2015. El trabajo aborda algunas de las principales aproximaciones y énfasis en el estudio de una realidad migratoria sobre la cual no existen aun consensos metodológicos, a través de un ejercicio de revisión de literatura especializada sobre la materia. El artículo desentraña algunas de las principales características, temáticas y prioridades en el estudio de la migración extraregional en tránsito por Centroamérica y sugiere algunas miradas futuras de interés para la comunidad académica, así como para los tomadores de decisiones en materia migratoria.
\end{abstract}

Palabras clave: migración en tránsito; Centroamérica; migración extraregional.

\begin{abstract}
The article examines the theoretical and conceptual complexity of migration in transit as a unit of analysis based on the case study of extraregional migratory flows in transit through Central America as of 2015. Through a review of specialized literature on the subject, the paper addresses some of the main approaches and emphasis on the study of a migratory reality upon which methodological consensus does not yet exist. The article unravels some of the main characteristics, themes and priorities in the study of extraregional migration in transit through Central America and suggests some future perspectives of interest to the academic community and immigration decisionmakers.
\end{abstract}

Keywords: migration in transit; Central America; extraregional migration.

Escuela de Relaciones Internacionales, Universidad Nacional de Costa Rica (UNA). Heredia, Costa Rica. Email: juan.mendez.barquero@una.cr. Orcid: https://orcid.org/0000-0001-9860-6788. 


\section{Introducción}

Este artículo tiene como objetivo central examinar la complejidad teóricoconceptual del concepto de 'migración en tránsito' como unidad de análisis a partir del caso de estudio de los flujos migratorios extraregionales en tránsito por Centroamérica, en particular, a partir de los acontecimientos que llevaron a un incremento de tales flujos en el año 2015.

Para ello, se emplea un ejercicio de revisión de literatura especializada en la última década en la materia, la cual, si bien no responde a la metodología de revisión sistemática, aspira a ser lo suficientemente exhaustivo para lograr evidenciar algunas de las principales características, temáticas y prioridades en el estudio de la migración extraregional en tránsito por Centroamérica.

Se utilizan principalmente fuentes secundarias como libros, capítulos de libros y artículos especializados que abordan distintas aristas, tanto del concepto de la migración en tránsito propiamente, como de los flujos de migración extraregional en tránsito por Centroamérica entre los años 2011 y 2020.

Se valoran e integran algunos de los principales trabajos teóricos, abordajes metodológicos, retos y discusiones a nivel internacional en materia de migración en tránsito, para luego derivar en los principales temas y énfasis sobre los cuales ha sido abordada de manera particular el fenómeno de la migración extraregional en tránsito por Centroamérica.

El artículo finaliza con algunas sugerencias de direcciones futuras para la comunidad académica y los tomadores de decisiones con base en lo discutido.

\section{Los flujos migratorios extraregionales en tránsito por Centroamérica como nueva realidad migratoria a partir del año 2015}

Si bien la migración extraregional en tránsito por Centroamérica no es un fenómeno reciente, hacia finales del año 2015, la región centroamericana experimentó una situación migratoria inédita cuando el Estado costarricense desarticuló una red criminal internacional dedicada al tráfico de personas, dejando a miles de migrantes extraregionales ${ }^{1}$ varados en territorio costarricense y panameño, con pocas posibilidades de abandonar la región de manera regular, dado que la amplia mayoría de estas personas habían ingresado a la región centroamericana de manera irregular y sin la documentación y requisitos migratorios requeridos.

Esta red criminal internacional se dedicaba especialmente al tráfico de personas provenientes de países en Asia, África y Cuba desde América del Sur en su ruta hacia Norteamérica a través del territorio centroamericano (Castillo, 2015).

\footnotetext{
1 Para efectos de este artículo se utilizará de aquí y en adelante el término 'migración extraregional' o 'migrantes extraregionales' como concepto genérico y amplio que incluye el movimiento de personas en tránsito por Centroamérica provenientes de países del Caribe, Asia y África. El término 'migración extracontinental' se entiende también en este artículo como sinónimo de 'migración extraregional'.
} 
A su vez, el Gobierno de Nicaragua, previendo una inminente crisis migratoria ante las noticas de acumulación de migrantes extraregionales varados en las fronteras costarricenses y panameñas, decide cerrar su frontera sur en noviembre de 2015, deportando hacia Costa Rica a cientos de ciudadanos cubanos y de otras nacionalidades que intentaban cruzar hacia ese país de manera irregular (Mata, 2016; Jiménez, 2016).

Semanas después, en diciembre de 2015, el Gobierno de Costa Rica toma la decisión de cerrar también su frontera sur con la finalidad de contener los flujos migratorios irregulares desde Panamá.

Durante las semanas que siguieron, autoridades costarricenses tuvieron que emitir un aproximado de ocho mil visas humanitarias (OIM, 2017), mayoritariamente a ciudadanos cubanos con la finalidad de regularizar su situación migratoria y permitirles libre movilidad en territorio nacional, al tiempo que se coordinaba con Gobiernos de la región una salida segura y ordenada ante una posible crisis migratoria.

Estos acontecimientos desencadenaron una suerte de 'efecto dominó' en la región, afectando principalmente a Costa Rica y Panamá, siendo este último país, un punto natural de ingreso a la región centroamericana, donde miles de migrantes extraregionales en tránsito provenientes de Cuba y de otras nacionalidades habían quedado igualmente varados y sin alternativas claras para continuar su trayectoria hacia Norteamérica² ${ }^{2}$.

Frente a la decisión de Nicaragua de no permitir el tránsito humanitario de migrantes extraregionales a través de su territorio a partir de noviembre de 2015, así como el anuncio de Costa Rica de no recibir más migración de ciudadanos cubanos y de otras nacionalidades a través de su frontera sur en marzo del año 2016, el Gobierno de Panamá toma la decisión igualmente de cerrar sus fronteras en mayo de ese mismo año a estas personas migrantes, quienes no contaban con la documentación necesaria para continuar su viaje a través de territorio centroamericano.

Tales medidas de contingencia fueron tomadas por los países centroamericanos concernidos ante un aumento exponencial en la aglomeración de personas migrantes extraregionales en tránsito, quienes se encontraban en su mayoría en una condición migratoria irregular. Esta fue una situación que, al criterio de las autoridades panameñas, estuvo cerca de "causar una crisis humanitaria" (Servicio Nacional de Migración, 2016).

Martínez y Clot (2018) argumentan que tales flujos migratorios extraregionales en 2015 desbordaron las capacidades institucionales de los países de tránsito, "lo cual ocasionó que cada país intentara evitar una crisis migratoria y humanitaria en su territorio" (p. 11).

2 Entre los principales países de origen de estos flujos migratorios extraregionales identificados por autoridades de Panamá entre los años 2009 y 2016 destacan Nepal, Congo, Bangladesh, Somalia, Ghana, China, Pakistán, Senegal y Eritrea (Servicio Nacional de Migración, 2016). 
Esta primera denominada 'oleada' de migrantes extraregionales varados en la región, en su mayoría migrantes en tránsito procedentes de Cuba, originó inclusive que la mayoría de los países centroamericanos implementaran medidas y estrategias diplomáticas, políticas, administrativas y humanitarias excepcionales a nivel interno y a nivel regional, con la finalidad de lograr una salida a tal situación.

Para diciembre del año 2015, los países centroamericanos (con la excepción de Nicaragua) habían acordado llevar a cabo puentes aéreos humanitarios, permitiendo el traslado de más de cinco mil migrantes cubanos, que habían quedado varados semanas atrás, desde Costa Rica hacia El Salvador, país desde el cual emprendieron un traslado terrestre hacia Guatemala y México, para llegar finalmente hasta la frontera con Estados Unidos (su destino original).

Sin embargo, una vez resuelta la denominada 'crisis de los migrantes cubanos', los países de la región tuvieron que afrontar casi de manera inmediata una segunda 'oleada' de migración en tránsito proveniente en su mayoría de países asiáticos, caribeños y africanos, quienes, al igual que las personas migrantes cubanas, habían quedado a la deriva en su travesía hacia Norteamérica cuando varias redes criminales fueron desarticuladas en el año 2015 por el Estado costarricense, seguido por el cierre de las fronteras de los países centroamericanos.

Sin la 'ayuda' de las redes criminales dedicadas al tráfico de personas, miles de migrantes extraregionales provenientes especialmente de países caribeños como Haití y otros países asiáticos y africanos, quedaron atrapados 3 en los sectores fronterizos de Costa Rica y Panamá por meses.

En este caso, a diferencia de los mecanismos regionales acordados para el abordaje de la situación de las personas migrantes cubanas, las autoridades de los países de la región, en especial Costa Rica y Panamá, no estuvieron en capacidad de brindar soluciones similares para el traslado de estas poblaciones hacia América del Norte, tales como puentes aéreos o terrestres, dadas las limitaciones económicas que estos grupos de migrantes extraregionales presentaban, los altos costos económicos y logísticos que implicaban tales operaciones; pero, más aún, debido a la condición migratoria irregular que estas personas mantenían para su ingreso a Estados Unidos o Canadá, a diferencia de los ciudadanos cubanos, quienes gozaban de privilegios migratorios especiales derivados de la legislación migratoria estadounidense.

Sin embargo, a pesar de que los flujos en tránsito de estas personas continuaron durante los siguientes meses -y años-, aunque en una menor intensidad, gracias a los esfuerzos de control de tales flujos y cuotas diarias establecidas entre países como Panamá y Costa Rica, la migración en tránsito de estas poblaciones migrantes

\footnotetext{
Morales (2020) utiliza el concepto de atrapados en el tránsito para hacer referencia a las "medidas también dirigidas a contener los flujos mixtos de una creciente migración extra regional y extracontinental de inmigrantes cubanos, haitianos, africanos y asiáticos" (p. 43) en su ruta por Centroamérica.
} 
extraregionales a través de la región centroamericana no ha cesado del todo, utilizando todo tipo de vías regulares e irregulares.

Los primeros estudios sobre esta dinámica migratoria identificaron factores como pobreza, desigualdad, abusos de derechos humanos, conflictos armados y todo tipo de persecución religiosa y social en sus países de origen como los motivos fundamentales para migrar (FLACSO, 2011, p. 16). Más de cinco años después, sin embargo, los flujos migratorios extraregionales en tránsito no han cesado completamente.

Datos de la Policía Profesional de Migración de Costa Rica registraron en el año 2018 un total de 8.961 personas migrantes en tránsito extraregionales y 19.628 personas durante el año 2019, mostrando un aumento de 119.03 por cierto (OIM, 2019b, p. 9); mientras que el Servicio Nacional de Migración de Panamá, país que constituye uno de los principales puntos de tránsito y registro en Centroamérica, registró en el año 2018 un aproximado de 9.065 entradas irregulares detectadas de personas provenientes de África, Asia, y el Caribe, número que ascendió a 18.179 en los primeros ocho meses del año 2019 (OIM, 2019b, p. 9).

En abril de 2019, autoridades costarricenses registraron el ingreso diario de más cien personas migrantes procedentes de países caribeños y otras regiones desde Panamá (Recio, 2019).

Durante el primer semestre del año 2020, autoridades migratorias de Costa Rica y Panamá continuaron registrando el tránsito de cientos personas migrantes extraregionales, provenientes especialmente de países como Haití, Congo, Bangladesh y Yemen (Pomareda, 2020), llegando el primero a recibir casi tres mil personas extraregionales tan solo en el mes de marzo de 2020 (OEA, 2020, p. 36) ${ }^{4}$.

\section{La construcción de la migración en tránsito como unidad de análisis: desafíos, brechas y abordajes contemporáneos}

Si bien los estudios sobre la migración en tránsito no son recientes, ciertamente el peso relativo y espacio que ha ocupado este tema dentro de la comunidad académica es menor, frente a otras temáticas o dimensiones de la migración internacional propiamente, tales como las causas subyacentes y factores de expulsión, la integración de personas migrantes, narrativas migratorias, entre muchas otras aristas del fenómeno migratorio. El estudio de la migración en tránsito pareciera que no forma parte de las prioridades y agendas de investigación en los principales centros de investigación en materia migratoria.

\footnotetext{
4 A diciembre del año 2020, la Dirección General de Migración y Extranjería de Costa Rica registró la emisión de casi cinco mil permisos de ingreso y transito (PITS) a personas migrantes extraregionales en su tránsito por territorio costarricense desde Panamá. Estas personas migrantes provienen de países como Angola, Bangladesh, Benin, Burkina Fasso, Camerun, Costa de marfil, Cuba, Eritrea, Etiopia, Gambia, Ghana, Guinea Bissau, Guyana francesa, Haiti, India, Irán, Líbano, Mali, Nepal, Nigeria y otros países africanos. Ver: Dirección General de Migración y Extranjería - DGME, 2020.
} 
Con algunas excepciones (Düvell, 2006; Düvell, 2012; Collyer, Düvell, de Haas, 2012), la mayoría de los trabajos sobre este tema se enmarcan en lo que llamaríamos estudios de caso, no así en el estudio y debate teórico sobre el concepto mismo de la migración en tránsito.

Así, esta materia ha sido abordada no de manera sistemática por la comunidad académica, sino de manera fragmentada a través de casos de estudio.

Por ejemplo, trabajos sobre el tránsito de migrantes a través de Europa (Düvell, Collyer, Molodikova, 2014), destacan los flujos migratorios en tránsito de países africanos y asiáticos y otras periferias de la Unión Europa a través de Rusia a partir de la desintegración de la antigua Unión Soviética en los años 1900 (Ivakhnyuk, 2014) y el tránsito de migrantes del medio oriente y otros países asiáticos a través de Turquía hacia la Unión Europea (İçduygu, Sert, 2014; Zijlstra, 2014).

En otras regiones, otros casos de estudio analizan las rutas y las dificultades que enfrentan flujos de migración centroamericana hacia Estados Unidos a través de México (Morales-Cardiel, 2016), la etnografía y espacios de dicha movilidad (Frank-Vitale, 2020), las necesidades particulares de personas refugiadas, solicitantes de refugio y asilo en tránsito por Indonesia (Missbach, 2017); o bien, de las personas migrantes o en necesidad de protección internacional del África subsahariana en Marruecos (Alioua, 2014; Collyer, 2010). Otros estudios de caso versan sobre la construcción política de país de tránsito y los abusos que se pueden cometer en países como Tailandia (Coddington, 2020).

En la región de las Américas, Guevara (2017) argumenta que el concepto de migración en tránsito ha sido discutido principalmente en el análisis de los procesos de migración irregular de ciudadanos centroamericanos hacia Estados Unidos, a través de territorio mexicano.

Todos estos estudios demuestran por un lado la necesidad de la comunidad académica de brindar aristas propias con base en casos puntuales, pero además demuestran que la migración en tránsito es un fenómeno global.

Si bien los enfoques y los temas centrales de estos y muchos otros trabajos son diversos y complejos, por ejemplo, los enfoques en la externalización de las fronteras y políticas de seguridad, el papel de la integración y la respuesta humanitaria de los gobiernos locales en la atención de flujos migratorios en tránsito (Larsen, Demir, Horvat, 2016), la mayoría de estos trabajos toman como punto de partida las realidades migratorias en tránsito hacia Europa.

Fargues (2009) sin embargo argumenta que este es un "nuevo término en la migración internacional que se refiere a personas inicialmente en ruta hacia regiones lejanas -Europa, América del Norte o Estados en el Golfo- quienes no completaron su trayecto porque no tenían los requerimientos de visa" (2009, p. 564).

Claramente, esta inclinación eurocéntrica se relaciona con el peso y magnitud de los enormes flujos migratorios en ruta hacia Europa que se han experimentado 
en las últimas décadas en ese continente, pero, además, en particular debido a las crisis de migrantes y refugiados (provenientes principalmente de Siria, Afganistán e Irak) que experimentó ese continente entre los años 2015 y 2016, catalogadas como de proporciones históricas por algunos (Theodorou, 2016).

Sin embargo, estos autores dan un paso más al afirmar que el concepto de migración en tránsito se utiliza casi de manera exclusiva en el contexto europeo para hacer referencia a los 'flujos irregulares'. Para Düvell, Molodikova y Collyer (2014, p. 13) 'transit migrant' (migrante en tránsito) se utiliza para referirse a individuos que han llegado a Europa.

A pesar de las limitaciones y posibles sesgos que estos autores pudieran tener en su abordaje, el valor de su propuesta recae en la visibilización de algunos de los principales temas que se identifican en esta literatura especializada: los asuntos humanitarios conexos, las estadísticas, asuntos legales, implicaciones geopolíticas, asuntos tecnológicos, entre otros.

Así, como categoría de análisis, el concepto de migración en tránsito es complejo, puede ser inclusive catalogado como controversial (Missbach, 2015) y despierta dudas sobre la mejor aproximación para su estudio. Esto debido en parte a las inconsistencias metodológicas que puede enfrentar el propio término; pero, además, porque el propio concepto puede estar vinculado a "formas particulares de conocimiento e imaginarios políticos, con implicaciones y consecuencias políticas específicas" (Basok, 2019, p. 85).

Düvell por ejemplo argumenta que el concepto de migración en tránsito es científicamente no muy claro y puede ser confundido con otras categorías tales como migración irregular y migración circular (Düvell, 2012 apud Collyer, Düvell, de Haas, 2012).

De los análisis más relevantes sobre los alcances y las limitaciones metodológicas que enfrenta el concepto de migración en tránsito, destacan los trabajos de Collyer et al. (2012), quienes agrupan algunos de los principales temas entorno a la migración en tránsito de la siguiente manera: zonas precarias de tránsito, personas atrapadas o varadas en sus rutas de tránsito y la situación de indeterminación legal a quienes se les rechaza la solicitud de asilo o refugio.

Las dificultades de definición de la migración en tránsito no son menores tampoco. Estos vacíos conceptuales podrían derivarse de las categorías migratorias propias en cada región o país, donde el elemento de voluntariedad o movilidad forzosa puede jugar algún papel, lo mismo el tema de los tiempos de estancias en los países de tránsito o las motivaciones del movimiento (económicas o seguridad).

Como bien apunta Missbach (2015), la migración en tránsito puede no denotar un tipo de migración, sino fases durante el proceso migratorio. Esta autora puntualiza que personas migrantes en tránsito pueden experimentar distintos tipos de migración en el tanto su estatus puede cambiar continuamente durante un periodo y lugar (Missbach, 2015, p. 118). 
Si bien el término migración o migrante en tránsito se ha mantenido en la literatura, no existe consenso aún dentro de la comunidad académica sobre su definición, alcances y dimensiones, si bien desde décadas atrás su uso se popularizó gracias al trabajo de ciertos organismos internacionales en el marco de las Naciones Unidas (Düvell, 2006).

Por ejemplo, para la Organización Internacional para las Migraciones (OIM), se entiende como tránsito una "parada de duración variable durante un viaje entre dos o más países" (OIM, 2019a, p. 236); entendiéndose aquí el concepto de país de tránsito como "un país por el que pasa una persona o grupo de personas, en cualquier viaje hacia el país de destino, o bien desde el país de destino hacia el país de origen o de residencia habitual" (OIM, 2019a, p. 162).

Asimismo, para este organismo internacional, el ciclo migratorio incluye etapas del proceso migratorio que comprenden la partida y "en algunos casos el tránsito a través de uno o varios países (...)" (OIM, 2019a, p. 34).

Para Papadopoulou-Kourkoula (citado en Guevara, 2017), la migración en tránsito se entiende como una "situación entre la emigración y el establecimiento que es caracterizado una estancia migratoria indefinida, legal o ilegal, y que puede desarrollarse o no en posterior migración dependiendo de una serie de factores estructurales e individuales" (párrafo 10).

Esta última definición complejiza aún más los alcances conceptuales y dimensiones analíticas de la migración en tránsito. Más aún, mientras la amplia mayoría de trabajos se refieren al concepto de migración en tránsito, otros autores hacen referencia a conceptos similares para referirse al mismo fenómeno migratorio, tales como el concepto de transmigrante (Casillas-Ramírez, 2011), transmigración (Acuña, 2019; Guevara, 2017), migración de paso (Casillas, 2020) y flujos mixtos de paso (Mora, Miranda, 2019; Mora-Izaguirre, 2020).

El cuadro 1 resume algunos de los principales conceptos utilizados como sinónimo de migración en tránsito en la literatura analizada:

Cuadro 1 - Algunos conceptos utilizados como sinónimo de migración en tránsito en la literatura especializada

\begin{tabular}{l|c|c}
\hline \multicolumn{1}{c|}{ Concepto } & Autor & Año \\
\hline Transmigrante & Casillas-Ramírez & 2011 \\
\hline Flujos migratorios mixtos & Flacso & 2011 \\
\hline Transmigration (inglés) & Guevara & 2017 \\
\hline Transmigración & Acuña & 2019 \\
\hline \multirow{2}{*}{ Flujos mixtos de paso } & Mora y Miranda & 2019 \\
\cline { 2 - 3 } & Mora-Izaguirre & 2020 \\
\hline Migración de paso & Casillas & 2020 \\
\hline
\end{tabular}

Nota: elaboración propia con base libros, capítulos de libros y artículos especializados. 
Por otro lado, paralelo a la diversidad conceptual que engloba el término de migración en tránsito, así como sus falencias metodológicas, inconsistencias y dificultades para identificar a este fenómeno como una unidad de análisis clara (Collyer et al., 2012; Düvell, 2006), otra subdimensión de la misma que surge de la revisión de literatura especializada, hace referencia al fenómeno de las poblaciones migrantes varadas o incapaces de seguir su trayectoria en países en tránsito.

Dentro de esta subdimensión, se puede identificar también un amplio abanico de temas, entre estos, las dificultades que enfrentan migrantes en tránsito varados cuando los países que pertenecen a un mismo bloque de integración regional (Unión Europea en este caso) tienen sistemas o modelos de recepción diferentes (Brekke, Brochmann, 2014); las condiciones de solicitantes de refugio y asilo que quedan varados en países que no cuentan con legislación apropiada (Missbach, 2019; Missbach, 2017); o bien, flujos mixtos varados por años en países en tránsito (Zijlstra, 2014).

En la literatura en inglés se utiliza mayoritariamente los términos 'stuck' (atascados) o 'stranded' (varados) indistintamente como sinónimos para hacer referencia a la misma situación, es decir, flujos de migración que por distintos motivos políticos, económicos, sociales y legales quedaron varados o atascados por tiempo definido o indefinido en países en tránsito donde no tenían pensado residir o permanecer.

Muchos de estos estudios se basan en análisis cualitativos y etnográficos llevados a cabo a través de entrevistas semiestructuradas aplicadas las personas migrantes y otros funcionarios en el terreno.

\section{La migración extraregional en tránsito por Centroamérica en la literatura especializada}

En Centroamérica, las agendas de investigación en materia migratoria son igualmente variadas y responden a prioridades institucionales y temáticas particulares.

Con la clara excepción de los flujos migratorios de ciudadanos centroamericanos en tránsito por territorio mexicano, el fenómeno de la migración en tránsito extraregional no ocupa tampoco un lugar central en los estudios migratorios, a diferencia, por ejemplo, de los amplios estudios sobre dinámicas de la migración laboral o bien, del impacto de las remesas en los países de origen.

Una parte importante de la literatura especializada existente responde a reportes, giras de campo e informes sobre las denominadas 'crisis' de la migración extraregional en tránsito por Centroamérica (Cortez, 2016a; OIM, 2016; OIM, 2019b).

Algunos otros estudios versan sobre cifras y tendencias (Rodríguez, 2016a) de los flujos de migración de personas migrantes centroamericanas por territorio mexicano y los retos para las políticas migratorias de los países en tránsito (Morales-Cardiel, 
2016), así como algunas prácticas de solidaridad por parte de las comunidades de acogida temporal a lo largo de sus trayectorias (Rodríguez, 2016b).

Guevara (2017) argumenta que la mayoría de la producción académica en materia de migración en tránsito se ha originado en los albores del siglo XX, siendo los últimos diez años el periodo en el que más trabajos se han desarrollado sobre el tema, especialmente a raíz de los denominados corredores migratorios y migración en tránsito de ciudadanos centroamericanos hacia Estados Unidos (a través de México).

No es casualidad que un amplio volumen de la literatura sobre la materia se dedique al análisis de las rutas clandestinas o irregulares (Martínez, Cobo, Narváez, 2015; Álvarez, 2009); dificultades humanitarias (Izcara, 2015); abusos, violaciones de derechos humanos y violencia en los trayectos (de la Torre, 2019; Yee, Torre, 2016; Aikin, Anaya, 2013; Morales, 2010; Ríos, 2014), y todo tipo de estudios sobre securitización de fronteras (Calleros, 2013), políticas públicas, implementadas en México para abordar los flujos migratorios de centroamericanos en ruta hacia Estados Unidos.

Un estudio de compilación bibliográfica especializada en migración en tránsito irregular entre los años 1990 y 2015, logró recabar 322 referencias, provenientes de 182 autores, 114 distintas instituciones, 44 revistas académicas y 24 países de publicación o realización, entre los que destaca México, Estados Unidos, países de Europa Occidental, Guatemala, Argentina y Turquía. Sobresalen en este trabajo temas como la migración en tránsito por México hacia Estados Unidos, con 254 registros y la migración en tránsito hacia otras regiones, con 68 registros (Rodríguez et al., 2016).

Otros trabajos aún más recientes (Eguren, Hernández, 2020) analizan fenómenos de migración en tránsito de ciudadanos centroamericanos hacia Estados Unidos bajo lo que ha sido denominado por la academia y la prensa como 'caravanas de migrantes'.

Este nuevo fenómeno migratorio de ciudadanos principales hondureños, guatemaltecos y salvadoreños en tránsito hacia los Estados Unidos entre los años 2018 y 2019, estimuló un nuevo interés y debate por comprender las motivaciones y características de tales movimientos, pero además otros análisis que buscan desentrañar los posibles vínculos y relaciones causales entre este fenómeno migratorio, los impactos adversos del cambio climático, la inseguridad, violencia organizada y, en particular, el papel que la sequía pudo haber jugado en los últimos años y su afectación directa en cultivos y granos esenciales como el maíz, los frijoles, el arroz y el café (CEPAL, 2018), en la decisión de migrar de estas personas.

En términos generales, en la región latinoamericana, muchos de los trabajos sobre migración en tránsito son empíricos y se sustentan en el análisis de casos de estudio, e involucran algún tipo de trabajo de campo o estudios etnográficos. 
Con relación a los flujos migratorios extraregionales propiamente, el grueso de la literatura especializada aborda el tema desde las particularidades propias de las rutas migratorias de ciudadanos del Caribe, países africanos y asiáticos a través de América del Sur, Centroamérica y Norteamérica.

Destacan algunos estudios particulares sobre el tránsito de migrantes haitianos a través de Ecuador (López Rivera, Wessel, 2017) y otros análisis sobre como la 'crisis migratoria' ocurrida en Centroamérica entre los años 2015 y 2016 desencadenaron una suerte de externalización de fronteras con repercusiones para el tránsito de migrantes de Cuba, Haití y otras nacionales en países como Ecuador (Herrera, Dalum, 2019).

Es importante resaltar que el abordaje de la migración extraregional en tránsito en la región latinoamericana se enmarca en muchas oportunidades dentro de informes, reportes y artículos sobre tráfico y trata de personas, así como dentro de trabajos sobre las dinámicas de las redes criminales que son contratadas por estas personas migrantes desde sus países de origen para completar su viaje hasta Norteamérica (Danelo, 2017).

Por ejemplo, Villalobos-Torres (2017) analiza las trayectorias, aspiraciones y motivaciones de personas migrantes en tránsito varadas en Costa Rica entre los años 2015 y 2016 dentro de un proyecto de investigación global que busca conocer las impresiones y nociones que tienen estas personas sobre el tema de trata y tráfico de personas.

Así, es de suponer que, en algunas oportunidades, el enfoque sobre la migración extraregional en tránsito se pierda o no sea tan evidente al encontrarse dentro de los trabajos sobre la materia de trata y tráfico de personas.

Los estudios, reportajes e informes sobre las denominadas crisis migratorias de los cubanos, haitianos y africanos (migrantes extraregionales en general) varados en Centroamérica entre los años 2015 y 2016, son naturalmente recientes, muchos de los cuales se concentran en describir las modalidades y rutas desde sus países de origen (Martínez, Clot, 2018), las dificultades enfrentadas durante los trayectos, la caracterización y tendencias de tales flujos (Mora, 2018, 2017; Villalobos-Torres, 2017).

Dada la novedad del fenómeno migratorio bajo estudio, especialmente a partir de los acontecimientos del año 2015, los estudios existentes son limitados, muchos de los cuales responden a informes y planes llevados a cabo por organismos internacionales como la Organización Internacional para las Migraciones (OIM).

Algunos de estos versan sobre propuestas o planes sobre la gobernanza regional de los flujos extraregionales (OIM, 2016), o bien sobre las tendencias y patrones de movilidad de estas poblaciones por la región centroamericana en los últimos años.

Otros trabajos se apoyan en estudios de casos, trabajo de campo y entrevistas a las propias personas migrantes varadas en su tránsito en países como Costa Rica o 
Panamá (Winters, Reichl, 2020; Winters, Mora, 2019; Winters, 2019), así como el tratamiento desde este fenómeno desde un enfoque sociológico de las corporalidades y prejuicios que se pudieron haber generado debido a los acontecimientos recientes en la región (Acuña, 2019).

Algunas investigaciones visibilizan las trayectorias y patrones de migración africana en América del Sur y el tránsito a través de Centroamérica en su ruta hacia América del Norte. Al criterio de Winters y Reiffen (2019) la gran mayoría de estas personas migrantes africanas han visto a Centroamérica como una zona de tránsito, quienes a su vez son vistas "como personas en tránsito, aunque a veces varadas, por los gobiernos, instituciones y habitantes centroamericanos" (p. 18).

\section{Reflexiones finales: algunos retos y direcciones futuras con relación a los estudios de la migración extraregional en tránsito por Centroamérica}

En términos generales y, como se desprende de la revisión de literatura especializada sobre migración en tránsito, se ha evidenciado por un lado retos y dificultades metodológicas sobre el propio concepto, pero, además, un abordaje fragmentado basado en estudios de caso, lo cual no resulta en sí mismo un problema, sino una realidad que la o el investigador interesado en la materia debe sortear y tener presente desde el inicio.

Los asuntos centrales que se abordan en la literatura especializada son igualmente diversos y abarcan temas no necesariamente interconectados, por ejemplo, externalización de fronteras, seguridad, respuestas locales, prácticas discursivas y otras subdimensiones como la migración en tránsito varada o atrapada.

Por otro lado, dada la novedad y las características del fenómeno de la migración extraregional en tránsito por Centroamérica a partir del año 2015, una parte importante de los estudios se han limitado describir tales flujos, mientras que otros se han valido de los enfoques etnográficos para profundizar en las experiencias de vida, dificultades y vejaciones que han sufrido en muchas ocasiones estas poblaciones migrantes en su travesía hacia América del Norte.

Así, si bien la literatura y los primeros estudios especializados sobre migración extraregional en tránsito por Centroamérica se pueden remontar una década atrás ${ }^{5}$, la comunidad académica aún se enfoca en la caracterización de las rutas utilizadas, perfiles, análisis etnográficos e historias de vida sobre trayectorias, retos, dificultades y modalidades de los flujos migratorios extraregionales desde sus países de origen, así como tendencias en el incremento de tales movimientos en los últimos años.

\footnotetext{
5 Por ejemplo, el primer "Diagnóstico sobre la situación actual, tendencias y necesidades de protección y asistencia de las personas migrantes y refugiadas extracontinentales en México y América Central" Ilevado a cabo en el año 2011 por la Facultad Latinoamericana de Ciencias Sociales (FLACSO) a solicitud de la Organización Internacional para las Migraciones (OIM) y la Agencia de las Naciones Unidas para los Refugiados (ACNUR) como apoyo a los trabajos y discusiones de los países miembros de la Conferencia Regional sobre Migración (CRM) (FLACSO, 2011).
} 
Todo lo anterior evidencia una clara complejidad para el análisis de la migración extraregional en tránsito por Centroamericana como objeto de estudio y unidad de análisis. El propio concepto de migración en tránsito extraregional, entendida en algunos contextos y por ciertos autores como migración extracontinental, pero, además, como "migraciones mixtas extracontinentales" (FLACSO, 2011), enfrenta un enorme reto al enmarcarse en algunas ocasiones dentro de estudios y análisis sobre trata y tráfico de personas.

Asimismo, a pesar de que el aumento en los flujos migratorios extraregionales en tránsito por Centroamérica ha generado impactos reales y visibles a nivel institucional en los países de la región en los últimos años, su abordaje integral por parte de la literatura resulta aún una tarea pendiente.

Por ejemplo, aún se carece de estudios que tengan como objetivo profundizar no solamente en las trayectorias y motivaciones macro o estructurales que impulsan a estas personas a migrar, sino que analicen como el tránsito de dichas poblaciones pueden generar nuevos escenarios de riesgo para sí mismas, o bien, replicar o ampliar escenarios de riesgo tanto en comunidades de acogida, como en los países de tránsito. Es decir, el establecimiento de una suerte de línea base en los países en tránsito que le permita a las y los tomadores de decisiones, direccionar de una manera más adecuada los recursos -limitados- con los cuales cuentan los países de tránsito para la atención de estas poblaciones migrantes, más allá de la asistencia humanitaria inmediata.

Estos estudios podrían abordar posibles cambios institucionales, nuevos enfoques de trabajo, implicaciones prácticas, operativas, políticas, financieras y sociales, tanto a nivel nacional, como a nivel local o municipal. Los alcances y enfoques pueden ser diversos, por ejemplo, mecanismos de gobernanza regional y transfronterizos, retos particulares que podrían enfrentar las poblaciones migrantes de conformidad con su nacionalidad o país de residencia, entre muchos otros. Se podría argumentar en este sentido que existe una necesidad manifiesta de abordar este fenómeno migratorio no solamente a partir de sus implicaciones teóricas, sino prácticas, dada la importancia de su peso relativo en la toma de decisiones para los países de la región.

Resulta además necesario fortalecer la apropiación del concepto de la migración en tránsito en los círculos académicos de la región, a partir de fenómenos o casos de estudio concretos como el que se analiza en este artículo, con la finalidad no solo de mejorar la respuesta institucional, sino con el propósito de evaluar si las aproximaciones teóricas y metodológicas son suficientes para lograr una justa compresión de este fenómeno.

La situación de inmovilidad de personas migrantes en tránsito (extraregionales) y sus necesidades particulares debe ser objeto además del más alto interés académico y político para los países concernidos. 
Con la excepción de los recientes informes e investigaciones llevados a cabo por organismos internacionales y algunos investigadores en Centroamérica y América del Sur sobre la materia, el interés sobre las particularidades e implicaciones teóricoprácticas de los flujos migratorios extraregionales en tránsito por Centroamérica, no forman parte aún de las agendas centrales de investigación de la región; menos aún, a partir de su abordaje desde la prospectiva, creación de escenarios o en materia de riesgos por ejemplo. Estos vacíos pueden ser subsanados con estudios posteriores en las temáticas indicadas arriba, especialmente cuando informes o trabajos recientes sobre patrones de migración en las Américas (Feldmann, Bada, Schutze, 2019) continúan soslayando y abordando de manera superficial estas dinámicas de migración extraregional en tránsito.

Futuras y potenciales direcciones de investigación deberían abordar además la situación de las personas migrantes extraregionales en tránsito que han dejado de serlo, es decir, los alcances y respuesta de la institucionalidad a nivel nacional y regional cuando estas personas deciden continuar su travesía y cruzar de un país a otro: ¿en que condición migratoria se encuentran?; ¿qué retos enfrentan?; iexisten mecanismos reales y complementarios de comunicación y cooperación entre gobiernos (país de origen y país de destino, por ejemplo) para dar seguimiento o monitorear la situación de estas personas?

Todas estas interrogantes pueden dar forma a futuros estudios en la materia. Tarea que no es fácil ciertamente, como bien apunta Missbach (2015), la migración en tránsito puede no denotar un tipo de migración, sino fases durante el proceso migratorio, lo cual significa que personas migrantes en tránsito pueden experimentar distintos tipos de migración en el tanto su estatus puede cambiar continuamente durante un periodo y lugar.

Finalmente, es importante recordar que el aumento en los flujos migratorios extraregionales por Centroamérica han sido una constante en el último lustro, de manera que su abordaje requiere una estrategia metodológica que tome en consideración no solamente las rutas y aspiraciones personales de las poblaciones migrantes mismas, sino un planteamiento teórico-práctico que contribuya a desentrañar las distintas etapas de la migración en tránsito, la evolución de las necesidades en el camino y las implicaciones para los actores locales, incluidas las comunidades de acogida.

\section{Referencias bibliográficas}

ACUÑA, Guillermo. Regímenes de corporalidad y recientes transmigraciones africanas en Costa Rica: Dispositivos y discursos sociales. Revista ÍTSMICA, n. 23, p. 103-118, Enero-junio 2019. DOI: https://doi.org/10.15359/istmica.23.9.

AIKIN, Olga; ANAYA, Alejandro. Crisis de derechos humanos de las personas migrantes en tránsito por méxico: redes y presión transnacional. Foro Internacional, v. 53, n. 1, p. 143-181, 2013. 
ALIOUA, Mehdi. Transnational Migration. The Case of Sub-Saharan Transmigrants Stopping Over in Morocco. In: DÜVELL, Frank; COLLYER, Michael; MOLODIKOVA, Irina (eds.). Transit Migration in Europe. Amsterdam: Amsterdam University Press, 2014, p. 79-98.

ÁLVAREZ, Soledad. Transitando en la clandestinidad: análisis de la migración indocumentada en tránsito por la frontera sur mexicana. Sistema de Información sobre Migraciones Andinas. Quito: Ecuador, 2009, p. 2-10.

BASOK, Tanya. The Discourse of "Transit Migration" in Mexico and Its "Blind Spot": Changing Realities and New Vocabularies. In: FELDMANN, Andreas E.; BADA, Xóchitl; SCHÜTZE, Stephanie (eds.). New Migration Patterns in the Americas. Challenges for the 21st Century. Cham: Palgrave Macmillan, 2019, p. 85-107.

BREKKE, Jan-Paul; Brochmann, Grete. Stuck in Transit: Secondary Migration of Asylum Seekers in Europe, National Differences, and the Dublin Regulation. Journal of Refugee Studies, v. 28, n. 2, 2014. DOI:10.1093/jrs/feu028.

CALLEROS, Juan Carlos. Seguridad pública y seguridad humana en la migración indocumentada de tránsito por México. Foro Internacional, v. LIII, n. 2, p. 317-336, 2013.

CASILLAS, Rodolfo. Migración internacional y cambio climático: conexiones y desconexiones entre México y Centroamérica. URVIO, Revista Latinoamericana de Estudios de Seguridad, n. 26, p. 73-92, enero-abril 2020.

CASILLAS-RAMÍREZ, Rodolfo. Central American Transmigrants Migratory Movement of Special Interest to Different Sectors within and outside Mexico. In: HOERDER, Dirk; FAIRES, Nora (eds.). Migrants and Migration in Modern North America. US: Duke University Press, 2011, p. 364-376.

CASTILLO, Diego. Policía desarticula banda internacional de tráfico de personas. La Nación, 10.11.2015. Disponible en: www.nacion.com.

CEPAL. Atlas migración en los países del norte de Centroamérica. Santiago: Comisión Económica para América Latina y el Caribe (CEPAL), 2018.

CODDINGTON, Kate. Producing Thailand as a transit country: borders, advocacy, and destitution. Mobilities, v. 15, n. 3, junio 2020. DOI: https://doi.org/10.1080/17450 101.2020.1759928.

COLLYER, Michael. Stranded Migrants and the Fragmented Journey. Journal of Refugee Studies, v. 23, n. 3, p. 273-293, 2010. DOI:10.1093/jrs/feq026.

COLLYER, Michael; DÜVELL, Frank; de HAAS, Hein. Critical Approaches to Transit Migration. Special Issue. Population, Space and Place, v. 18, n. 4, p. 407-481, July/ August 2012.

CORTEZ, Charlene. Tendencia de la migración cubana y su impacto en la región centroamericana. Revista Latinoamericana de Derechos Humanos, Número monográfico extraordinario, 2016a, p. 145-167.

CORTEZ, Charlene. Tendencias de la migración intrarregional en Centroamérica. Revista Latinoamericana de Derechos Humanos, Número monográfico extraordinario, 2016b, p. 125-143. 
CRM. Cuarta reunión del grupo de trabajo ad-hoc sobre migrantes extra-regionales [documento técnico]. 2017. Disponible en: <https://portal.crmsv.org/es/eventos/ iv-reunion-grupo-ad-hoc-migrantes-extra-regionales $>$.

DANELO, David. People smuggling in Latin America. In: COYNE, John; NYST, Madeleine. Strategy. People smugglers globally. Australia: Australian Strategic Policy Institute, 2017, p. 53-56.

de la TORRE, Renée. La transmigración en México: el cruce de los caminos de la fe y los derechos humanos. In: GIMÉNEZ, Verónica (comp.). La religión ante los problemas sociales: Espiritualidad, poder y sociabilidad en América Latina. Buenos Aires: CLACSO, 2019, p. 229-258. DOI: 10.2307/j.ctvnp0jnq.12.

Dirección General de Migración y Extranjería - DGME. Informes Estadísticos Anuales 2020. 2020. Disponible en: https://www.migracion.go.cr/.

DÜVELL, Franck. Crossing the fringes of Europe: Transit migration in the EU's neighbourhood. Working Paper No. 33, University of Oxford, 2006. Disponible en: <https://www.compas.ox.ac.uk/wp-content/uploads/WP-2006-033-Düvell_ Fringe_Migration.pdf $>$.

DÜVELL, Franck. Transit migration: A blurred and Politicised Concept. Population, Space and Place. Special Issue Papers, v. 18, n. 4, p. 415-427, 2012. DOI: https:// doi.org/10.1002/psp.631.

DÜVELL, Frank; COLLYER, Michael; MOLODIKOVA, Irina (eds.). Transit Migration in Europe. Amsterdam: Amsterdam University Press, 2014.

EGUREN, Joaquín; HERNÁNDEZ, Sindy (eds.). Caravanas de migrantes: manifestaciones de la compleja realidad Centroamericana. Colección OBIMID, v. 6. Madrid: Universidad Pontificia Comillas, 2020.

FARGUES, Philippe. Work, Refuge, Transit: An Emerging Pattern of Irregular Immigration South and East of the Mediterranean. The International Migration Review, v. 43, n. 3, p. 544-577, Fall 2009.

FELDMANN, Andreas; BADA, Xóchitl; SCHUTZE, Stephanie (eds.). New migration patterns in The Americas. Challenges for the 21st Century. Switzerland: Palgrave Macmillan, 2019.

FLACSO. Diagnóstico sobre la situación actual, tendencias y necesidades de protección y asistencia de las personas migrantes y refugiadas extracontinentales en México y América Central. San José: Facultad Latinoamericana de Ciencias Sociales (FLACSO), 2011.

FRANK-VITALE, Amelia. Stuck in Motion: Inhabiting the Space of Transit in Central American Migration. The Journal of Latin American and Caribbean Anthropology, v. 25, n. 1, p. 67-83, 2020. DOI: 10.1111/jlca.12465.

GUEVARA, Yaatsil. "Transmigration." InterAmerican Wiki: Terms - Concepts Critical Perspective, 2017. Disponible en: <www.uni-bielefeld.de/cias/wiki/t_ Transmigration.html>.

HERRERA, Gioconda; DALUM, Ulla. "Migration crises" and humanitarism in Latina America: the case of Ecuador. Danish Institute for International Studies, 2019, p. 32-49. 
IÇDUYGU, Ahmet; SERT, Deniz. Migrants' Uncertainties versus the State's Insecurities Transit Migration in Turkey. In: DÜVELL, Frank; COLLYER, Michael; MOLODIKOVA, Irina (eds.). Transit Migration in Europe. Amsterdam: Amsterdam University Press, 2014, p. 37-54.

IVAKHNYUK, Irina. The East-to-West Circuit Transit Migration through Russia. In: DÜVELL, Frank; COLLYER, Michael; MOLODIKOVA, Irina (eds.). Transit Migration in Europe. Amsterdam: Amsterdam University Press, 2014, p. 127-151.

IZCARA, Simon Pedro. Los transmigrantes centroamericanos en México. Latin American Research Review, v. 50, n. 4, p. 49-68, 2015.

JIMÉNEZ, Eillyn. Africanos se agolpan en la frontera norte de Peñas Blancas. La Nación, 12.07.2016. Disponible en: < https://www.nacion.com/el-pais/politica/africanos-seagolpan-en-la-frontera-norte-de-penas-blancas/MZNVGZYDQRFNZGS6DNTK2AT $\mathrm{DHE} /$ story/>.

LARSEN, Maren; DEMIR, Ema; HORVAT, Maja. Migrant and refugee transit: the role of local authorities in humanitarian response. International Institute for Environment and Development, Issue date September 2016.

LÓPEZ RIVERA, Andrés; WESSEL, Jana. Migración haitiana en tránsito por ecuador. Comentario Internacional: Revista del Centro Andino de Estudios Internacionales, v. 17, p. 19-32, 2017. DOl: http://dx.doi.org/10.32719/2631254 9.2017.17.1.

MARTÍNEZ, Germán; CLOT, Jen. La “odisea” de los migrantes cubanos en América: modalidades, rutas y etapas migratorias. Revista pueblos y fronteras digital, v. 13, p. 1-27, 2018.

MARTíNEZ, Graciela; COBO, Salvador; NARVÁEZ, Juan Carlos. Trazando rutas de la migración de tránsito irregular o no documentada por México. Perfiles latinoamericanos, v. 23, n. 45, p. 127-155, 2015.

MATA, Esteban. Canciller tico: gobiernos veían para para otro lado mientras redes de tráfico cruzaban a cubanos por Centroamérica. La Nación, 16.11.2016. Disponible en: <https://www.nacion.com/el-pais/politica/canciller-tico-gobiernos-veian-paraotro-lado-mientras-redes-de-trafico-cruzaban-a-cubanos-por-centroamerica/3S2B LG46DNHHTAV5L55YLRPP34/story/>.

MISSBACH Antje. Troubled Transit: Asylum Seekers Stuck in Indonesia. ISEAS - Yusof Ishak Institute, 2015.

MISSBACH, Antje. Troubled Transit: Asylum Seekers Stuck in Indonesia. Journal of Refugee Studies, v. 30, n. 4, p. 628-630, 2017. DOI: https://doi.org/10.1093/jrs/ fex031.

MISSBACH, Antje. Asylum Seekers' and Refugees' Decision-Making in Transit in Indonesia: The Need for In-depth and Longitudinal Research. Bijdragen tot de Taal-, Land-en Volkenkunde, v. 175, n. 4, p. 419-445, 2019.

MORA, Cynthia. De Brasil hasta Canadá: el paso de los extracontinentales por Costa Rica. In: MORA, Cynthia (coord.). Migraciones en Costa Rica: un fenómeno histórico y dinámico desde diversas perspectivas disciplinares. San José, Costa Rica: FLACSO, 2017, p. 175-203. 
MORA, Cinthya. El paso de los Africanos por Costa Rica: retos estatales de una zona de paso. In: ALCÁNTARA, Manuel; GARCÍA, Mercedes; SÁNCHEZ, Francisco (coords.). Migraciones. Memoria del 56 Congreso Internacional de Americanistas. Salamanca, 2018.

MORA, Cinthya; MIRANDA, Fabiola. Flujos mixtos de paso por Centroamérica (2015-2017) y la respuesta estatal costarricense: acciones similares, omisiones y coordinaciones con otros países del área. In: CIDICER. Construcciones Identitarias e Imaginarios Sociales. San José: Universidad de Costa Rica, 2019, p. 178-192.

MORA-IZAGUIRRE, Cynthia. La marcha de los flujos mixtos por Costa Rica a la luz de algunas teorías que describen las migraciones del S XXI. REMHU, Revista Interdisciplinar da Mobilidade Humana, Brasília, v. 28, n. 60, p. 95-108, dez. 2020.

MORALES, Abelardo. Migraciones internacionales, refugiados y desplazamientos internos en Centroamérica: factores de riesgo e instrumentos para fortalecer la protección de los derechos humanos. Informe de Investigación. Sexto Informe Estado de la Región. San José: Estado de la Región, 2020.

MORALES, Julio. Violencia y derechos humanos de centroamericanos en méxico: la paradoja entre ser un país de migrantes y un país de tránsito. Boletín Americanista, n. 60, p. 227-246, 2010.

MORALES-CARDIEL, Jorge. La inmigración indocumentada por países de tránsito, nuevo paradigma de los sistemas migratorios: el caso de México y Marruecos. Perspectiva Geográfica, v. 21, n. 2, 2016.

Naciones Unidas. OIM colabora en el plan para los migrantes cubanos varados en Costa Rica. Noticias ONU, 12.01. 2016a. Disponible en: < https://news.un.org/es/ story/2016/01/1348471>.

Naciones Unidas; Sale de Costa Rica último grupo de cubanos varados con ayuda de la OIM. Noticias ONU, 15.03.2016b. Disponible en: < https://news.un.org/es/ story/2016/03/1352911>.

OEA. Costa Rica: caracterización y análisis de las políticas en materia de migración internacional y refugiados. Washington D.C: OAS Cataloging-in-Publication Data, 2020 .

OIM. Glosario de la OIM sobre Migración. Organización Internacional para las Migraciones OIM, 2019a.

OIM. Migración extraregional en Sudamérica y Mesoamérica: perfiles, experiencias y necesidades. San José: OIM, $2019 b$.

OIM. Plan para fortalecer la gobernanza de los flujos de migrantes extrarregionales en Mesoamérica. San José: OIM, 2016.

OIM. Marco estratégico binacional Costa Rica-Panamá 2017-2019. Marco Operacional en Situaciones de Crisis Migratoria. San José: OIM, 2017.

POMAREDA, Fabiola. Migrantes extracontinentales continúan cruzando por Paso Canoas en la frontera sur. Seminario, 09.06.2020. Disponible en: <https:// semanariouniversidad.com/pais/migrantes-extracontinentales-continuan-cruzandopor-paso-canoas-en-la-frontera-sur/>. 
RCM. Grupo Ad Hoc sobre Migrantes Extra-Continentales. Regional Conference on Migration, 2016. Disponible en: <http://www.crmsv.org/es/eventos/grupo-adhoc-sobre-migrantes-extra-continentales $>$.

RECIO, Patricia. Nueva oleada de migrantes: unas 100 personas atraviesan Costa Rica cada día. La Nación, 15.04.2019. Disponible en: <https:/www. nacion.com/el-pais/servicios/nueva-oleada-de-migrantes-trae-al-pais-hasta-10/ RUBC7P53AJGJ7ABKSS2KELIDS4/story/>.

RÍOS, Juana Luisa. La violencia en la migración de tránsito de centroamericanos en México. Clivajes. Revista de Ciencias Sociales, v. 1, n. 1, p. 1-17, 2014.

RODRÍGUEZ, Ernesto. Migración centroamericana en tránsito irregular por México: nuevas cifras y tendencias. CANAMID Policy Brief Series, PB14. Guadalajara, México: CIESAS, 2016a. Disponible en: <www.canamid.org>.

RODRÍGUEZ, Ernesto; FERNÁNDEZ, Claudia; LUNA, Rubén; RODRÍGUEZ, Edyam. Bibliografía sobre migración en tránsito irregular. México, Ciudad de México: Instituto Tecnológico Autónomo de México, 2016.

RODRÍGUEZ, María Tereza. Migración en tránsito y prácticas de ayuda solidaria en el centro de Veracruz, México 1. Encuentro, n. 103, p. 47-58, $2016 b$.

Servicio Nacional de Migración. Flujo Migratorio de Extracontinentales y Cubanos por la República de Panamá. [power point slides]. 2016. Disponible en: < https://portal. crmsv.org/es/eventos/iv-reunion-grupo-ad-hoc-migrantes-extra-regionales $>$.

THEODOROU, Maria. Migration/Refugee Crisis: A Challenge of Historic Proportions for Europe. Proceedings of the Annual Meeting. American Society of International Law, v. 110, p. 178-181, 2016.

VILLALOBOS-TORRES, Gabriela. El tránsito de migrantes por Costa Rica: el caso de las personas cubanas que persiguen el «sueño americano. Revista Espiga, v. 16, n. 34, p. 197-214, 2017. DOI: http://dx.doi.org/10.22458/re.v17i34.1800.

WINTERS, Nanneke. Haciendo-lugar en tránsito. reflexión sobre la migración africana y trabajo de campo en Darién, Panamá. REMHU, Reevista. Interdisciplinar da Mobilidade Humana, Brasília, v. 27, n. 56, p. 235-243, ago. 2019.

WINTERS, Nanneke; MORA, Cynthia. Es cosa suya: entanglements of border externalization and African transit migration in northern Costa Rica. Comparative Migration Studies, v. 7, n. 27, 2019. DOI: https://doi.org/10.1186/s40878-0190131-9.

WINTERS, Nanneke; REICHL, Elena. Pay and go? Transit Migration regimes and migrant navigation in Central America. 2020. Disponible en: <https://www.law. ox.ac.uk/research-subject-groups/centre-criminology/centreborder-criminologies/ blog/2020/04/pay-and-go>.

WINTERS, Nanneke; REIFFEN, Franziska. Haciendo-lugar vía huellas y apegos: las personas migrantes africanas y sus experiencias de movilidad, inmovilidad e inserción local en América Latina. REMHU, Reevista. Interdisciplinar da Mobilidade Humana, Brasília, v. 27, n. 56, p. 11-33, ago. 2019. DOI: <http://dx.doi. org/10.1590/1980-85852503880005602>. 
YEE, José Carlos; TORRE, Eduardo. Lidiando con la Frontera Vertical: Estrategias Migratorias de los Hondureños en Tránsito por México. REMHU, Revista Interdisciplinar da Mobilidade Humana, v. 24, n. 47, p. 97-114, 2016.

ZIJLSTRA, Judith. Stuck on the Way to Europe? Iranian Transit Migration to Turkey. Insight Turkey, v. 16, n. 4, p. 183-199, 2014. 